\title{
Distributed control for cooperative hyperbolic systems involving Schrödinger operator
}

\author{
A. H. Qamlo
}

Received: 12 February 2013 / Revised: 24 February 2013 / Accepted: 26 February 2013 / Published online: 13 March 2013

(C) The Author(s) 2013. This article is published with open access at Springerlink.com

\begin{abstract}
In this paper, some hyperbolic systems involving Schrödinger operator defined on $R^{n}$ have been established. The existence and uniqueness for the state of these systems have been proved, Then the necessary and sufficient conditions of optimality for such systems have been obtained by a set of equations and inequalities.
\end{abstract}

Keywords Cooperative hyperbolic systems .

Optimal control · Schrödinger operator .

Distributed control problem

\section{Mathematics Subject Classification 49 J 20}

\section{Introduction}

The necessary and sufficient conditions of optimality for systems governed by elliptic, parabolic, and hyperbolic operators have been studied by Lions in $[7,8]$. The considered systems in these problems are in the scalar case (system of one equation).

The discussion is extended to $2 \times 2$ systems for example in $[1,5,9,10,12]$ and to $n \times n$ systems in [11].

Optimal control problem for systems involving Schrödinger operators has been studied for the following elliptic system of distributed type [10]:

$$
\begin{cases}(-\Delta+q) y_{1}=a y_{1}+b y_{2}+f_{1} & \text { in } R^{n}, \\ (-\Delta+q) y_{2}=c y_{1}+d y_{2}+f_{2} & \text { in } R^{n}, \\ y_{1}, y_{2} \rightarrow 0 \text { as }|\mathrm{x}| \rightarrow \infty . & \end{cases}
$$

and for parabolic system of boundary type in [1].

A. H. Qamlo $(\varangle)$

Mathematics Department, Faculty of Applied sciences, Umm Al-Qura University, P.O. Box 1348, Makkah 21955, Saudi Arabia

e-mail: drahqamlo@yahoo.com
Systems with different potentials and positive weight function is studied in [12] and with variable coefficients is studied in [9].

The existence of optimal control for systems like (1) has been proved with $q(x)=0$ in [6], and for semi linear cooperative systems in [5].

Time-optimal control problem for cooperative hyperbolic systems involving the Laplace operator is studied in [2].

Here, we consider the following $2 \times 2$ cooperative hyperbolic systems involving Schrödinger operator:

$$
\left\{\begin{array}{l}
\frac{\partial^{2} y_{1}(x)}{\partial t^{2}}+(-\Delta+q) y_{1}=a y_{1}+b y_{2}+f_{1}(x, t) \text { in } Q \\
\frac{\partial^{2} y_{2}(x)}{\partial t^{2}}+(-\Delta+q) y_{2}=c y_{1}+d y_{2}+f_{2}(x, t) \text { in } Q \\
y_{1}, y_{2} \rightarrow 0 \text { as }|\mathrm{x}| \rightarrow \infty \\
\left.y_{1}\right|_{\Sigma}=\left.y_{2}\right|_{\Sigma}=0, \\
y_{1}(x, 0)=y_{1,0}(x), \quad y_{2}(x, 0)=y_{2,0}(x) \quad \text { in } R^{n} \\
\frac{\partial y_{1}(x, 0)}{\partial t}=y_{1,1}(x), \quad \frac{\partial y_{2}(x, 0)}{\partial t}=y_{2,1}(x) \quad \text { in } R^{n}
\end{array}\right.
$$

with

$$
\begin{aligned}
y_{1}, y_{2} \in & L^{2}\left(0, T ; V_{q}\left(R^{n}\right)\right), \\
& \frac{\partial y_{1}}{\partial t}, \frac{\partial y_{2}}{\partial t} \in L^{2}\left(0, T ; V_{q}^{\prime}\left(R^{n}\right)\right) .
\end{aligned}
$$

where

$a, b, c$ and $d$ are given numbers such that $b, c>0$,

(in this case, we say that the system (2) is cooperative)

$q(\mathrm{x})$ is a positive function and tending to $\infty$ at infinity,

and $\left.Q=R^{n} \times\right] 0, T[$ with boundary $\Sigma=\Gamma \times] 0, T[$.

We first prove the existence and uniqueness of the state for these systems, then we introduce the optimal control of distributed type for these systems. 


\section{Some concepts and results}

In this paper, we shall consider some results introduced in [3], [10] concerning the eigenvalue problem

$$
\begin{cases}(-\Delta+q) \phi=\lambda(q) \phi \quad \text { in } & R^{n} \\ \phi(x) \rightarrow 0 \text { as } \quad|x| \rightarrow \infty, & \phi \succ 0\end{cases}
$$

The associated variational space is $V_{q}\left(R^{n}\right)$, the completion of $D\left(R^{n}\right)$, with respect to the norm:

$$
\|y\|_{q}=\left(\int_{R^{n}}\left[|\nabla y|^{2}+q|y|^{2}\right] d x\right)^{1 / 2}
$$

Since the imbedding of $V_{q}\left(R^{n}\right)$ in to $L^{2}\left(R^{n}\right)$ is compact, then the operator $(-\Delta+q)$ considered as an operator in $L^{2}\left(R^{n}\right)$ is positive self-adjoint with compact inverse. Hence its spectrum consists of an infinite sequence of positive eigenvalues, tending to infinity; moreover the smallest one which is called the principal eigenvalue denoted by $\lambda(q)$ is simple and is associated with an eigenfunction which does not change sign in $R^{n}$. It is characterized by:

$$
\lambda(q) \int_{R^{n}}|y|^{2} d x \leq \int_{R^{n}}\left[|\nabla y|^{2}+q|y|^{2}\right] d x \quad \forall y \in V_{q}\left(R^{n}\right) .
$$

We have the following embedding :

$$
\begin{aligned}
& V_{q}\left(R^{n}\right) \times V_{q}\left(R^{n}\right) \subseteq L^{2}\left(R^{n}\right) \times L^{2}\left(R^{n}\right) \\
& \quad \subseteq V_{q}^{\prime}\left(R^{n}\right) \times V_{q}^{\prime}\left(R^{n}\right)
\end{aligned}
$$

which is continuous and compact .

Let us introduce the space $L^{2}\left(0, T ; V_{q}\left(R^{n}\right)\right)$ of measurable function $t \rightarrow f(t)$ which is defined on open interval $(0, T)$, since the variable $t \in(0, T)$ and $T<\infty$ denotes the time .

On $(0, T)$ with Lebesgue measure $d t$ we have the norm:

$$
\|f(t)\|_{L^{2}\left(0, T ; V_{q}\left(R^{n}\right)\right)}=\left(\int_{(0, T)}\|f(t)\|_{V_{q}\left(R^{n}\right)}^{2} d t\right)^{1 / 2} \prec \infty
$$

and the scalar product

$$
(f(t), g(t))_{L^{2}\left(0, T ; V_{q}\left(R^{n}\right)\right)}=\int_{(0, T)}(f(t), g(t))_{V_{q}\left(R^{n}\right)} d t,
$$

the space $L^{2}\left(0, T ; V_{q}\left(R^{n}\right)\right)$ with the scalar product and the norm above is a Hilbert space .

Analogously, we can define the spaces $L^{2}\left(0, T ; L^{2}\left(R^{n}\right)\right)$ $=L^{2}(Q)$, with the scalar product

$$
\begin{aligned}
(f(t), g(t))_{L^{2}(Q)} & =\int_{(0, T)}(f(t), g(t))_{L^{2}\left(R^{n}\right)} d t \\
& =\int_{Q} f(t) \cdot g(t) d x d t
\end{aligned}
$$

then we have the following embedding

$\left(L^{2}\left(0, T ; V_{q}\left(R^{n}\right)\right)\right)^{2} \subseteq\left(L^{2}(Q)\right)^{2} \subseteq\left(L^{2}\left(0, T ; V_{q}^{\prime}\left(R^{n}\right)\right)\right)^{2}$

\section{Existence and uniqueness of solution}

We introduce the bilinear form

$\pi(t ; y, \psi)=(A(t) y, \psi)\left(L^{2}\left(R^{n}\right)\right)^{2}$,

$y=\left\{y_{1}, y_{2}\right\}, \quad \psi=\left\{\psi_{1}, \psi_{2}\right\} \in\left(V_{q}\left(R^{n}\right)\right)^{2}$,

$A(t) y \in\left(V_{q}^{\prime}\left(R^{n}\right)\right)^{2}$

where

$$
\begin{aligned}
& A(t) y(x)=\left\{(-\Delta+q) y_{1}-a y_{1}-b y_{2},\right. \\
& \left.(-\Delta+q) y_{2}-c y_{1}-d y_{2}\right\}
\end{aligned}
$$

then

$$
\begin{aligned}
\pi(t ; y, \psi)= & \frac{1}{b} \int_{R^{n}}\left[\nabla y_{1} \nabla \psi_{1}+q y_{1} \psi_{1}\right] d x \\
& +\frac{1}{c} \int_{R^{n}}\left[\nabla y_{2} \nabla \psi_{2}+q y_{2} \psi_{2}\right] d x \\
& -\int_{R^{n}} y_{1} \psi_{2} d x-\frac{d}{c} \int_{R^{n}} y_{2} \psi_{2} d x \\
& -\frac{a}{b} \int_{R^{n}} y_{1} \psi_{1} d x-\int_{R^{n}} y_{2} \psi_{1} d x .
\end{aligned}
$$

For all $y, \psi \in\left(V_{q}\left(R^{n}\right)\right)^{2}$, the function $t \rightarrow \pi(t ; y, \psi)$ is measurable on $(0, T)$.

By using the necessary and sufficient conditions for having the maximum principle and existence of positive solutions for cooperative system (1) which have been obtained by Fleckinger [4] and take the form

$\left\{\begin{array}{l}a \prec \lambda(q), d \prec \lambda(q), \\ (\lambda(q)-a)(\lambda(q)-d) \succ b c,\end{array}\right.$

the coerciveness condition of the bilinear form (8) in $\left(V_{q}\left(R^{n}\right)\right)^{2}$ has been proved by Serag [10], that means

$\pi(t ; y, y) \geq C\left(\left\|y_{1}\right\|_{q, m}^{2}+\left\|y_{2}\right\|_{q, m}^{2}\right), \quad C \succ 0$ 
Theorem 1 Under the hypotheses (3) and (10), if $f_{1}, f_{2} \in$ $L^{2}\left(0, T ; V_{q}^{\prime}\left(R^{n}\right)\right), y_{1,0}(x), y_{2,0}(x) \in V_{q}\left(R^{n}\right)$ and $y_{1,1}(x), y_{2,1}$ $(x) \in V_{q}^{\prime}\left(R^{n}\right)$, then there exists a unique solution $y=$ $\left\{y_{1}, y_{2}\right\} \in\left(L^{2}\left(0, T ; V_{q}\left(R^{n}\right)\right)\right)^{2}$ for system (2).

Proof Let $\psi \rightarrow L(\psi)$ be a continuous linear form defined on $\left(L^{2}(Q)\right)^{2}$ by

$$
\begin{aligned}
L(\psi)= & \frac{1}{b} \int_{Q} f_{1}(x, t) \psi_{1}(x) d x d t \\
& +\frac{1}{c} \int_{Q} f_{2}(x, t) \psi_{2}(x) d x d t \\
& +\frac{1}{b} \int_{R^{n}} y_{1,1}(x) \psi_{1}(x, 0) d x \\
& +\frac{1}{c} \int_{R^{n}} y_{2,1}(x) \psi_{2}(x, 0) d x \\
\forall \psi= & \left\{\psi_{1}, \psi_{2}\right\} \in\left(L^{2}\left(0, T ; V_{q}\left(R^{n}\right)\right)\right)^{2},
\end{aligned}
$$

then by Lax-Milgram lemma, there exists a unique element $y=\left\{y_{1}, y_{2}\right\} \in\left(L^{2}\left(0, T ; V_{q}\left(R^{n}\right)\right)\right)^{2}$ such that

$$
\begin{aligned}
& \frac{1}{b}\left(\frac{\partial^{2} y_{1}}{\partial t^{2}}, \psi_{1}\right)+\frac{1}{c}\left(\frac{\partial^{2} y_{2}}{\partial t^{2}}, \psi_{2}\right)+\pi(t ; y, \psi)=L(\psi) \\
& \forall \psi=\left\{\psi_{1}, \psi_{2}\right\} \in\left(L^{2}\left(0, T ; V_{q}\left(R^{n}\right)\right)\right)^{2},
\end{aligned}
$$

Now, let us multiply both sides of first equation of system (2) by $\frac{1}{b} \psi_{1}(x)$, and the second equation by $\frac{1}{c} \psi_{2}(x)$ then integration over $\mathrm{Q}$, we have:

$$
\begin{aligned}
& \frac{1}{b} \int_{Q}\left[\frac{\partial^{2} y_{1}(x)}{\partial t^{2}}+(-\Delta+q) y_{1}-a y_{1}-b y_{2}\right] \psi_{1} d x d t \\
& =\frac{1}{b} \int_{Q} f_{1}(x, t) \psi_{1} d x d t, \\
& \frac{1}{c} \int_{Q}\left[\frac{\partial^{2} y_{2}(x)}{\partial t^{2}}+(-\Delta+q) y_{2}-c y_{1}-d y_{2}\right] \psi_{2} d x d t \\
& =\frac{1}{c} \int_{Q} f_{2}(x, t) \psi_{2} d x d t .
\end{aligned}
$$

By applying Green's formula:

$$
\begin{aligned}
& \frac{1}{b} \int_{Q} \frac{\partial^{2} y_{1}(x)}{\partial t^{2}} \psi_{1}(x) d x+\frac{1}{b} \int_{Q} \nabla y_{1} \nabla \psi_{1} d x d t \\
& -\frac{1}{b} \int_{\Sigma} \psi_{1} \frac{\partial y_{1}}{\partial v_{A}} d \Sigma-\frac{1}{b} \int_{R^{n}} \psi_{1}(x, 0) \frac{\partial y_{1}(x, 0)}{\partial t} d x \\
& +\int_{Q}\left(\frac{q}{b} y_{1}-\frac{a}{b} y_{1}-y_{2}\right) \psi_{1} d x d t
\end{aligned}
$$

$$
\begin{aligned}
= & \frac{1}{b} \int_{Q} f_{1}(x, t) \psi_{1} d x d t, \\
& \frac{1}{c} \int_{R^{n}} \frac{\partial^{2} y_{2}(x)}{\partial t^{2}} \psi_{2}(x) d x+\frac{1}{c} \int_{Q} \nabla y_{2} \nabla \psi_{2} d x d t \\
& -\frac{1}{c} \int_{\Sigma} \psi_{2} \frac{\partial y_{2}}{\partial v_{A}} d \Sigma-\frac{1}{c} \int_{R^{n}} \psi_{2}(x, 0) \frac{\partial y_{2}(x, 0)}{\partial t} d x \\
& +\int_{Q}\left(\frac{q}{c} y_{2}-y_{1}-\frac{d}{c} y_{2}\right) \psi_{2} d x d t \\
= & \frac{1}{c} \int_{Q} f_{2}(x, t) \psi_{2} d x d t .
\end{aligned}
$$

By sum the two equations, then comparing the summation with (8), (11) and (12) we get:

$$
\begin{aligned}
& -\frac{1}{b} \int_{\Sigma} \psi_{1} \frac{\partial y_{1}}{\partial v_{A}} d \Sigma-\frac{1}{c} \int_{\Sigma} \psi_{2} \frac{\partial y_{2}}{\partial v_{A}} d \Sigma \\
& -\frac{1}{b} \int_{R^{n}} \psi_{1}(x, 0) \frac{\partial y_{1}(x, 0)}{\partial t} d x-\frac{1}{c} \int_{R^{n}} \psi_{2}(x, 0) \frac{\partial y_{2}(x, 0)}{\partial t} d x \\
& =\frac{1}{b} \int_{R^{n}} y_{1,1}(x) \psi_{1}(x, 0) d x+\frac{1}{c} \int_{R^{n}} y_{2,1}(x) \psi_{2}(x, 0) d x,
\end{aligned}
$$

then we deduce that:

$$
\begin{aligned}
& \left.y_{1}\right|_{\Sigma}=\left.y_{2}\right|_{\Sigma}=0 \\
& \frac{\partial y_{1}(x, 0)}{\partial t}=y_{1,1}(x), \quad \frac{\partial y_{2}(x, 0)}{\partial t}=y_{2,1}(x) \quad \text { in } \quad R^{n} .
\end{aligned}
$$

which completes the proof.

\section{Formulation of the control problem}

The space $L^{2}(Q) \times L^{2}(Q)$ is the space of controls. For a control $u=\left\{u_{1}, u_{2}\right\} \in\left(L_{2}(Q)\right)^{2}$, the state $y(u)=$ $\left\{y_{1}(u), y_{2}(u)\right\} \in\left(L^{2}\left(0, T ; V_{q}\left(R^{n}\right)\right)\right)^{2}$ of the system (2) is given by the solution of

$$
\left\{\begin{array}{l}
\frac{\partial^{2} y_{1}(u)}{\partial t^{2}}+(-\Delta+q) y_{1}(u)=a y_{1}(u)+b y_{2}(u)+f_{1}+u_{1} \text { in } Q, \\
\frac{\partial^{2} y_{2}(u)}{\partial t^{2}}+(-\Delta+q) y_{2}(u)=c y_{1}(u)+d y_{2}(u)+f_{2}+u_{2} \text { in } Q \\
y_{1}, y_{2} \rightarrow 0 \text { as }|\mathrm{x}| \rightarrow \infty \\
\left.y_{1}(u)\right|_{\Sigma}=\left.y_{2}(u)\right|_{\Sigma}=0 \\
y_{1}(x, 0, u)=y_{1,0}(x), \quad y_{2}(x, 0, u)=y_{2,0}(x) \text { in } R^{n}, \\
\frac{\partial y_{1}(x, 0, u)}{\partial t}=y_{1,1}(x), \quad \frac{\partial y_{2}(x, 0, u)}{\partial t}=y_{2,1}(x) \quad \text { in } R^{n} .
\end{array}\right.
$$

with

$$
\begin{aligned}
& y_{1}(u), y_{2}(u) \in L^{2}\left(0, T ; V_{q}\left(R^{n}\right)\right), \\
& \frac{\partial y_{1}(u)}{\partial t}, \frac{\partial y_{2}(u)}{\partial t} \in L^{2}\left(0, T ; V_{q}^{\prime}\left(R^{n}\right)\right)
\end{aligned}
$$


The observation equation is given by $z(u)=\left\{z_{1}(u), z_{2}(u)\right\}$ $=y(u)=\left\{y_{1}(u), y_{2}(u)\right\}$.

For a given $z_{d}=\left\{z_{d 1}, z_{d 2}\right\} \in\left(L^{2}(Q)\right)^{2}$, the cost function is given by

$$
\begin{aligned}
J(v)= & \left\|y_{1}(v)-z_{d 1}\right\|_{L^{2}(Q)}^{2}+\left\|y_{2}(v)-z_{d 2}\right\|_{L^{2}(Q)}^{2} \\
& +(N v, v){ }_{\left(L^{2}(Q)\right)^{2}}
\end{aligned}
$$

where $N \in L\left(\left(L^{2}(Q)\right)^{2},\left(L^{2}(Q)\right)^{2}\right)$ is a Hermitian positive definite operator:

$$
(N u, u)_{\left(L^{2}(Q)\right)^{2}} \geq \gamma\|u\|_{\left(L^{2}(Q)\right)^{2}}^{2}, \quad \gamma \succ 0 .
$$

The control problem then is to find $u=\left\{u_{1}, u_{2}\right\} \in U_{a d}$ such that $J(u) \leq J(v)$,

where $U_{a d}$ is a closed convex subset of $\left(L^{2}(Q)\right)^{2}$.

Since the cost function (14) can be written as (see [7]):

$$
J(v)=a(v, v)-2 L(v)+\left\|y(0)-z_{d}\right\|_{\left(L^{2}(Q)\right)^{2}}^{2},
$$

where $a(v, v)$ is a continuous coercive bilinear form and $L(v)$ is a continuous linear form on $\left(L^{2}(Q)\right)^{2}$. Then using the general theory of Lions [7], there exists a unique optimal control $u \in U_{a d}$ such that $J(u)=\inf J(v)$ for all $v \in U_{a d}$. Moreover, we have the following theorem which gives the necessary and sufficient conditions of optimality :

Theorem 2 Assume that (10) and (15) hold. If the cost function is given by (14), the optimal control $u=\left\{u_{1}, u_{2}\right\} \in$ $\left(L_{2}(Q)\right)^{2}$ is then characterized by the following equations and inequalities:

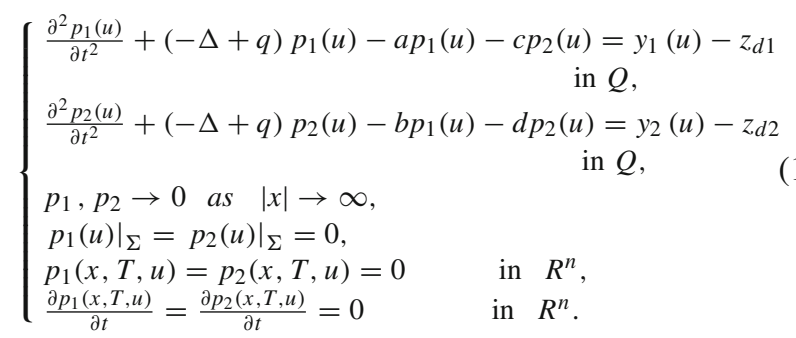

with

$$
\begin{aligned}
& p_{1}(u), p_{2}(u) \in L^{2}\left(0, T ; V_{q}\left(R^{n}\right)\right), \\
& \quad \frac{\partial p_{1}(u)}{\partial t}, \frac{\partial p_{2}(u)}{\partial t} \in L^{2}\left(0, T ; V_{q}^{\prime}\left(R^{n}\right)\right) \\
& \left(p_{1}(u), v_{1}-u_{1}\right)_{L^{2}(Q)}+\left(p_{2}(u), v_{2}-u_{2}\right)_{L^{2}(Q)} \\
& +(N u, v-u)_{\left(L^{2}(Q)\right)^{2}} \geq 0 \quad \forall v=\left\{v_{1}, v_{2}\right\} \in U_{a d}
\end{aligned}
$$

together with (13), where $p(u)=\left\{p_{1}(u), p_{2}(u)\right\}$ is the adjoint state .
Proof The optimal control $u=\left\{u_{1}, u_{2}\right\} \in\left(L_{2}(Q)\right)^{2}$ is characterized by (see[7])

$$
J \prime(u)(v-u) \geq 0 \quad \forall v \in U_{a d},
$$

which is equivalent to:

$$
\begin{aligned}
& \left(y(u)-z_{d}, y(v)-y(u)\right)\left(L^{2}(Q)\right)^{2} \\
& +(N u, v-u)\left(L^{2}(Q)\right)^{2} \geq 0
\end{aligned}
$$

i.e.

$$
\begin{aligned}
& \left(y_{1}(u)-z_{d 1}, y_{1}(v)-y_{1}(u)\right)_{L^{2}(Q)} \\
& \quad+\left(y_{2}(u)-z_{d 2}, y_{2}(v)-y_{2}(u)\right)_{L^{2}(Q)} \\
& \quad+(N u, v-u)\left(L^{2}(Q)\right)^{2} \geq 0
\end{aligned}
$$

this inequality can be written as

$$
\begin{aligned}
& \int_{0}^{T}\left(y_{1}(u)-z_{d 1}, y_{1}(v)-y_{1}(u)\right)_{L^{2}\left(R^{n}\right)} d t \\
& +\int_{0}^{T}\left(y_{2}(u)-z_{d 2}, y_{2}(v)-y_{2}(u)\right)_{L^{2}\left(R^{n}\right)} d t \\
& \quad+(N u, v-u)_{\left(L^{2}(Q)\right)^{2}} \geq 0
\end{aligned}
$$

Now, since

$$
\begin{aligned}
& (p, B y)\left(L^{2}(Q)\right)^{2} \\
& =\int_{0}^{T}\left(p_{1}(u), \frac{\partial^{2} y_{1}(u)}{\partial t^{2}}+(-\Delta+q) y_{1}(u)\right. \\
& \left.\quad-a y_{1}(u)-b y_{2}(u)\right)_{L^{2}\left(R^{n}\right)} d t \\
& +\int_{0}^{T}\left(p_{2}(u), \frac{\partial^{2} y_{2}(u)}{\partial t^{2}}+(-\Delta+q) y_{2}(u)\right. \\
& \left.\quad-c y_{1}(u)-d y_{2}(u)\right)_{L^{2}\left(R^{n}\right)} d t
\end{aligned}
$$

where

$$
\begin{aligned}
& B y(u)=B\left\{y_{1}(u), y_{2}(u)\right\} \\
& =\left\{\frac{\partial^{2} y_{1}(u)}{\partial t^{2}}+(-\Delta+q) y_{1}(u)-a y_{1}(u)-b y_{2}(u),\right. \\
& \left.\quad \frac{\partial^{2} y_{2}(u)}{\partial t^{2}}+(-\Delta+q) y_{2}(u)-c y_{1}(u)-d y_{2}(u)\right\}
\end{aligned}
$$

by using Green formula and (13), we get

$$
\begin{aligned}
& (p, B y)\left(L^{2}(Q)\right)^{2} \\
& =\int_{0}^{T}\left(\frac{\partial^{2} p_{1}(u)}{\partial t^{2}}+(-\Delta+q) p_{1}(u)\right.
\end{aligned}
$$




$$
\begin{aligned}
& \left.-a p_{1}(u)-c p_{2}(u), y_{1}(u)\right)_{L^{2}\left(R^{n}\right)} d t \\
& +\int_{0}^{T}\left(\frac{\partial^{2} p_{2}(u)}{\partial t^{2}}+(-\Delta+q) p_{2}(u)-b p_{1}(u)\right. \\
& \left.-d p_{2}(u), y_{2}(u)\right)_{L^{2}\left(R^{n}\right)} d t \\
& =\left(B^{*} p, y\right)_{\left(L^{2}(Q)\right)^{2}}
\end{aligned}
$$

then

$$
\begin{aligned}
& B^{*} p(u)=B^{*}\left\{p_{1}(u), p_{2}(u)\right\} \\
& =\left\{\frac{\partial^{2} p_{1}(u)}{\partial t^{2}}+(-\Delta+q) p_{1}(u)-a p_{1}(u)-c p_{2}(u),\right. \\
& \left.\frac{\partial^{2} p_{2}(u)}{\partial t^{2}}+(-\Delta+q) p_{2}(u)-b p_{1}(u)-d p_{2}(u)\right\}
\end{aligned}
$$

and

$$
\begin{aligned}
& A^{*} p(u)=A^{*}\left\{p_{1}(u), p_{2}(u)\right\} \\
& =\left\{(-\Delta+q) p_{1}(u)-a p_{1}(u)-c p_{2}(u)\right. \\
& \left.\quad(-\Delta+q) p_{2}(u)-b p_{1}(u)-d p_{2}(u)\right\}
\end{aligned}
$$

since the adjoint equation takes the form:

$\frac{\partial^{2} p(u)}{\partial t^{2}}+A^{*} p(u)=y(u)-z_{d}$

and from Theorem1, we get a unique solution $p(u) \in$ $\left(L^{2}\left(0, T ; V_{q}\left(R^{n}\right)\right)\right)^{2}$ which satisfies

$$
\begin{aligned}
p_{1}(u), p_{2}(u) & \in L^{2}\left(0, T ; V_{q}\left(R^{n}\right)\right), \\
& \frac{\partial p_{1}(u)}{\partial t}, \frac{\partial p_{2}(u)}{\partial t} \in L^{2}\left(0, T ; V_{q}^{\prime}\left(R^{n}\right)\right) .
\end{aligned}
$$

This proves system (16).

Now, we transform (18) by using (16) as follows:

$$
\begin{aligned}
& \int_{0}^{T}\left(\frac{\partial^{2} p_{1}(u)}{\partial t^{2}}+(-\Delta+q) p_{1}(u)-a p_{1}(u)\right. \\
& \left.\quad-c p_{2}(u), y_{1}(v)-y_{1}(u)\right)_{L^{2}\left(R^{n}\right)} d t \\
& +\int_{0}^{T}\left(\frac{\partial^{2} p_{2}(u)}{\partial t^{2}}+(-\Delta+q) p_{2}(u)-b p_{1}(u)\right. \\
& \left.\quad-d p_{2}(u), y_{2}(v)-y_{2}(u)\right)_{L^{2}\left(R^{n}\right)} d t \\
& +(N u, v-u)\left(L^{2}(Q)\right)^{2} \geq 0 .
\end{aligned}
$$

Using Green formula, we obtain

$$
\begin{aligned}
& \left.\int_{0}^{T} \int_{1}(u),\left(\frac{\partial^{2}}{\partial t^{2}}+(-\Delta+q)\right) y_{1}(v)-y_{1}(u)\right)_{L^{2}\left(R^{n}\right)} d t \\
& +\int_{0}^{T}-a\left(p_{1}(u), y_{1}(v)-y_{1}(u)\right)_{L^{2}\left(R^{n}\right)} d t \\
& +\int_{0}^{T}-c\left(p_{2}(u), y_{1}(v)-y_{1}(u)\right)_{L^{2}\left(R^{n}\right)} d t \\
& +\int_{0}^{T}\left(p_{2}(u),\left(\frac{\partial^{2}}{\partial t^{2}}+(-\Delta+q)\right)_{2}(v)-y_{2}(u)\right)_{L^{2}\left(R^{n}\right)} d t \\
& +\int_{0}^{T}-b\left(p_{1}(u), y_{2}(v)-y_{2}(u)\right)_{L^{2}\left(R^{n}\right)} d t \\
& +\int_{0}^{T}-d\left(p_{2}(u), y_{2}(v)-y_{2}(u)\right)_{L^{2}\left(R^{n}\right)} d t \\
& +(N u, v-u)_{\left(L^{2}(Q)\right)^{2} \geq 0 .}
\end{aligned}
$$

Using (13), we have

$$
\begin{aligned}
& \int_{0}^{T}\left(p_{1}(u), v_{1}-u_{1}\right)_{L^{2}\left(R^{n}\right)} d t \\
& +\int_{0}^{T}\left(p_{2}(u), v_{2}-u_{2}\right)_{L^{2}\left(R^{n}\right)} d t \\
& +(N u, v-u)_{\left(L^{2}(Q)\right)^{2} \geq 0}
\end{aligned}
$$

which is equivalent to

$$
\begin{gathered}
\left(p_{1}(u), v_{1}-u_{1}\right)_{L^{2}(Q)}+\left(p_{2}(u), v_{2}-u_{2}\right)_{L^{2}(Q)} \\
+(N u, v-u)_{\left(L^{2}(Q)\right)^{2} \geq 0}
\end{gathered}
$$

Thus the proof is complete.

Acknowledgments The author would like to express his gratitude to Professor H. M. Serag, Mathematics Department, Faculty of Sciences, AL-Azhar University for suggesting the problem and critically reading the manuscript.

Open Access This article is distributed under the terms of the Creative Commons Attribution License which permits any use, distribution, and reproduction in any medium, provided the original author(s) and the source are credited. 


\section{References}

1. Bahaa GM (2006) Boundary control for cooperative parabolic systems governed by Schrodinger operator. Differ Equ Control Process 1:79-88

2. El-Saify HA, Serag HM, Shehata MA (2009) Time optimal control problem for cooperative hyperbolic systems involving the Laplace operator. J Dyn Control Syst 15(3):405-423

3. Fleckinger J (1981) Estimates of the number of eigenvalues for an operator of Schrödinger type. Proc R Soc Edinb Sect A 89(34):355-361

4. Fleckinger J (1994) Method of sub-suber solutions for some elliptic system defined on $R^{n}$. Universite Toulouse, Preprint UMR MIP 3

5. Fleckinger J, Serag HM (1995) Semi linear cooperative elliptic systems on $R^{n}$. Rend Math Appl 5(1):89-108
6. Gali IM, Serag HM (1995) Optimal control of cooperative elliptic systems defined on $R^{n}$. J Egypt Math Soc 3:33-39

7. Lions JL (1971) Optimal control of systems governed by partial differential equations. Springer, Berlin

8. Lions JL (1981) Some methods in the mathematical analysis of systems and their control. Science Press, Beijing

9. Serag HM (1998) On optimal control for elliptic systems with variable coefficients. Rev Math Appl 19:37-41

10. Serag HM (2000) Distributed control for cooperative systems governed by Schrödinger operator. J Discret Math Sci Cryptogr 3(13):227-234

11. Serag HM (2004) Optimal control of systems involving Schrödinger operators. Int J Control Intell Syst 32(3):154-157

12. Serag HM, Qamlo AH (2005) On elliptic systems involving Schrödinger operators. Mediterr J Meas Control 1(2):91-96 